Evaluar.

2004, n ${ }^{\circ} 4$ (setiembre)
Laboratorio de Evaluación Psicológica y Educativa. Facultad de Psicología Universidad Nacional de Córdoba (Argentina).ISSN N ${ }^{\circ}$ 1667-4545

\title{
Diagnóstico de Habilidades Básicas para el ingreso a primer grado en niños de zonas urbano-marginales y rurales.
}

\author{
Furlan, L.* \& Alderete, A.* \\ * Laboratorio de Evaluación Psicológica y Educativa. Universidad Nacional de Córdoba
}

\begin{abstract}
Resumen: En el presente trabajo se sintetiza la experiencia realizada en cinco escuelas rurales de la provincia de Córdoba con el propósito de articular la investigación aplicada al desarrollo de tecnología (adecuación de una prueba de diagnostico escolar) y la capacitación de un grupo de docentes. Se utilizó una prueba elaborada para el diagnóstico de habilidades básicas para el ingreso a primer grado destinada a evaluar niños pertenecientes a sectores urbano marginados (DIPUM), con el objetivo de analizar su adecuación para evaluar niños de sectores pobres rurales. Por otro lado, se capacitó a docentes en diagnóstico y evaluación de las habilidades básicas y planificación del proceso de aprendizaje en el primer grado. Se comparó el rendimiento de los niños de zona rural con una muestra de niños de zona urbana y se realizó un estudio de la validez predictiva de las puntuaciones del DIPUM. Los resultados mostraron diferencias significativas entre los grupos y escasa validez predictiva. Si bien la aplicación de la prueba fue valorada positivamente por los docentes y utilizada para el aprestamiento de los niños, los resultados indican que deberían realizarse nuevos estudios técnicos antes de ser utilizada como diagnóstico en niños de sectores rurales.
\end{abstract}

Palabras claves: evaluación-diagnóstico- educación preescolar- habilidades básicas.

\section{Introducción}

El presente trabajo sintetiza la experiencia que realizamos en cinco escuelas rurales de las sierras de Córdoba que tuvo el propósito de articular la investigación aplicada al desarrollo de tecnología (adecuación de una prueba de diagnostico escolar) y la capacitación en servicio de un grupo de docentes.

Para ello, trabajamos con el DIPUM, un instrumento construido para evaluar a niños de poblaciones urbano-marginadas que inician la escolaridad primaria (Alderete, 
1997). El mismo nos permite conocer el desarrollo de habilidades perceptivo-motrices y cognoscitivas a través de un conjunto de ítems cuyas consignas y materiales resultan próximos a la cotidianeidad de estos sectores sociales. Se pretenden salvar así, los sesgos socio culturales producidos al evaluar con pruebas acordes a patrones evolutivos de clases medio-altas o poblaciones de otros países.

Tenemos evidencias que el DIPUM puede ser empleado en el sector urbano marginado de Córdoba Capital (San Millán y Pauletti, 1993). No obstante, para su aplicación a niños de condición humilde que habitan en las serranías se requiere un estudio previo a fin de analizar la capacidad de evaluar, a través de sus ítems, las habilidades de niños que si bien son de condición humilde, se han criado en un contexto diferente al estudiado al diseñar la prueba.

También quisimos estudiar la validez predictiva de la prueba, es decir la capacidad del DIPUM para predecir el rendimiento escolar de los niños en el primer trimestre de clases.

Al mismo tiempo, trabajamos con las maestras para capacitarlas en los fundamentos y modo de uso del DIPUM, la interpretación de la información que aporta y el diseño de actividades áulicas que (a partir del diagnostico de cada niño) les faciliten el desarrollo de habilidades escasamente logradas.

Las docentes aportaron datos y reflexiones relevantes que permitieron profundizar nuestro conocimiento de las problemáticas comunes a las escuelas de la zona (grados múltiples, heterogeneidad socio cultural, actividades de subsistencia, etc.) como así también de las diferencias existentes entre las mismas escuelas, ligadas a su ubicación geográfica y a las poblaciones que atienden.

La evaluación es una actividad esencial en las prácticas educativas y juega un papel de significación tanto para los alumnos como para los docentes y la institución escolar. Permite obtener un conjunto de datos que retroalimentan el constante proceso de toma de decisiones inherente a la tarea del maestro.

Al inicio de la escolaridad primaria, es necesario contar con un diagnóstico de las habilidades perceptivo-motrices y cognitivas con las que el niño cuenta para enfrentar con éxito las tareas de aprendizaje correspondientes al primer grado, ya que si bien el desarrollo evolutivo sigue pautas generales, el ritmo con que cada niño va adquiriendo estas habilidades, es variable respecto de otros de su misma edad. 
Estas diferencias son explicables a partir de la singular manera en que los factores biológico-madurativos y socio-culturales interactúen en la historia de cada niño, por lo que es de esperar que en un grupo de escolares los niveles sean heterogéneos, aún cuando hayan cursado el Jardín de Infantes.

Considerar esta variabilidad de puntos de partida puede aumentar la eficacia de las acciones educativas, si se construyen dispositivos de aprendizaje aprovechables a partir del bagaje de experiencias previas que traen los diferentes niños y no se supone una homogeneidad inexistente.

Por otra parte, si bien existen ciertos parámetros de normalidad para el desarrollo infantil, cada niño lo expresa de un modo idiosincrásico que refleja las características del entorno familiar y social con el que interactúa cotidianamente.

Este entorno, puede ser bastante diferente al escolar, sobre todo en familias con escaso acceso a la instrucción formal, o cuyas formas de subsistencia requieren de conocimientos prácticos que se adquieren informalmente, por transmisión de una generación a otra y como parte de un proceso global de socialización. Ejemplos de estos conocimientos en el ámbito rural son los referentes al cuidado de animales, la ordeña, el reconocimiento de hierbas, la fabricación de pan, etc..

Por consiguiente, nos interrogamos acerca de si ¿las diferencias culturales son reconocidas como tales o toman el carácter de deficiencias en relación a un parámetro de desarrollo esperable en los sectores de población al que pertenecen los docentes y demás profesionales?

Con frecuencia ocurre que la dificultades que los niños villeros o campesinos tienen para adaptarse al trabajo escolar (o la dificultad de la escuela para diseñar formas de trabajo acordes a los estilos de aprendizaje de estos niños) se significan de modo tal, que se pone en duda la normalidad de su desarrollo. Estos "déficits" se suelen atribuir a la escasa estimulación que los niños reciben de sus padres y la forma de abordarla se expresa en la creencia de que lo mas adecuado puede ser la derivación a una institución de "educación especial".

Para evaluar el rendimiento escolar se recomienda el uso de las pruebas denominadas con referencia a criterio, que nos informan de la posición de un individuo en relación a un dominio conductual definido explícitamente, y de este modo cobra un significado más claro cualquier cambio de la posición del sujeto en el contexto de ese 
dominio, pues refleja un cambio interpretable en términos conductuales y no solo la variación comparada con las posiciones relativas a otros sujetos (característica de las pruebas por normas). En consecuencia nos permiten conocer qué sabe hacer el alumno y qué no. (Himmel,1979).

Esto es relevante para el carácter formativo de la evaluación porque al medir cambios en los individuos, es decir diferencias intraindividuales o grupales como efecto del proceso educativo, podemos adecuar la acción educativa.

Uno de los aspectos fundamentales a analizar es la validez de la prueba, que se refiere a tres aspectos diferentes: de contenido, conceptual y predictiva. En este caso estudiaremos su validez predictiva.

En síntesis, los objetivos de esta investigación fueron:

- Analizar la validez predictiva de los puntajes de la Prueba de Habilidades Básicas para el ingreso a primer grado, en el contexto de las Escuelas Rurales.

- Capacitar a los docentes a través de su participación en una experiencia de investigación aplicada.

- Comparar el desempeño en la prueba de niños pertenecientes al sector urbano marginado y niños pertenecientes al sector pobre rural.

\section{EI Fracaso escolar en los sectores económicamente desfavorecidos}

De modo coincidente, diversos autores como Juan Carlos Tedesco (1981), Justa Ezpeleta (1991) y Luis Heredia (1996), señalan que los mayores índices de abandono y repitencia se encuentran en los sectores de la población económicamente desfavorecidos, siendo el ámbito rural donde esta realidad se acentúa aún más.

Si bien el Sistema Educativo ha extendido su cobertura hacia los sectores pobres, permitiéndoles el acceso al mismo, las elevadas cifras del fracaso (deserción y repitencia) nos indican que el aprovechamiento del servicio no ha sido el esperado y resulta necesaria una profunda revisión de las estrategias diseñadas para atender las necesidades educativas de estos sectores de la población.

Al analizar las causas de este fenómeno, Heredia (1996) indica que a pesar de que las mismas son tanto endógenas como exógenas al sistema, es frecuente encontrar una depositación de la responsabilidad del fracaso en el alumno y su falta de gusto por el estudio. Esta visión es asumida incluso, por muchos de los alumnos y pareciera 
exceptuar a la escuela de su papel determinante en la producción del problema, y de la consecuentemente necesidad de revisión crítica de su accionar.

Nos interesa profundizar especialmente en algunos de los factores que contribuyen al fracaso, por ejemplo, las diferencias entre la cultura escolar y la de las comunidades a las que pertenecen los niños, sus implicancias en el trabajo áulico y la relación educativa y la preparación del docente para enfrentarse a una realidad sociocultural y un tipo de trabajo que pueden ser bastante diferentes a los que conoció en su propia historia de escolarización y su formación del magisterio.

Creemos que la diversidad cultural ha sido escasamente considerada en el diseño de las ofertas educativas destinadas a los sectores pobres rurales y urbanos. Bajo el supuesto de la necesidad de brindar una formación homogénea, se han administrado los mismos conocimientos a personas con intereses, motivaciones y proyectos distintos, tomando como referentes los de los sectores medios y urbanos de la población.

Las políticas de regionalización apuntan a revertir esta modalidad, pero aún son incipientes.

Según Heredia (1996) "el divorcio con el contexto social al que pertenece es un rasgo común en la escuela pública, que se profundiza en el medio pobre rural y urbano-marginal por el choque socio cultural que representan la cultura oficial (textos escritos, hábitos, discurso, etc) y la cultura de los usuarios".

Un concepto que nos permite comprender más profundamente este fenómenos es el de Estilos Educativos, es decir "las modalidades que revisten las acciones de transmisión y construcción de los distintos saberes comunitarios, en otras palabras, las estrategias culturales de producción, transmisión y apropiación del conocimiento"'(Heredia, 1996)

Cuando un niño accede a la escuela, debido a su socialización primaria, trae consigo cierta forma de percibir el mundo, como así también ciertos estilos para adquirir conocimiento, a partir de los que intentará dar significación a las nuevas experiencias e informaciones que la escuela le va ofreciendo.

Descubrir estos estilos de aprender para instrumentarlos en la enseñanza no es una tarea fácil, pero sí necesaria, si se concibe el proceso educativo en términos de compartir significados. Si el esfuerzo por comprender al otro es realizado solamente por 
el alumno socio-culturalmente marginado, las probabilidades de entablar un vínculo productivo son bastante escasas.

El autor considera también el proceso de interpretación del comportamiento del otro, inherente a la relación educativa, y en el que hay un proceso de mutua adjudicación de sentido a las actos que cada uno realiza. Al respecto señala que: "cuando las distancias culturales son muy marcadas se tiende a considerar el comportamiento del otro, su experiencia, como no normal. Nos erigimos, de la manera más honesta imaginable en los paradigmas de la normalidad. Consideramos sinceramente que nuestra habla es normal, que nuestro modo de pensar es normal, que nuestros sentimientos son normales, que nuestra moral es normal, etc..."

No es de extrañar que estas interpretaciones estén fundamentando la creencia de que un niño necesita ser derivado a una escuela especial en tanto posee algún grado de "anormalidad"

Por otra parte debemos considerar que en el interior de "lo rural" coexisten una gran variedad de experiencias y expectativas respecto del rendimiento escolar de los niños. Cada familia participa desde cierto lugar en los procesos productivos y esta ubicación se relaciona con sus aspiraciones de progreso en el sistema educativo formal, como así también con los estilos que desarrolle para la educación de los hijos.

Los mismos comprenden cierta modalidad de comunicación, particularmente en lo relativo a la "estructura participatoria". Este concepto, desarrollado por Susan Phillips (citada por Ogbu 1984), se refiere a "una constelación de normas, derechos, y obligaciones que determinan la percepción de los participantes de lo que está pasando en el intercambio comunicativo e influye en el resultado de la comunicación y el aprendizaje". También señala que "cuando la estructura participatoria del salón de clases se correspondía con la estructura participatoria de la comunidad a la que los educandos pertenecen el rendimiento era bueno y que cuando la estructura participatoria que imponía el maestro no se correspondía con la vernácula de los alumnos, el rendimiento de estos caía sensiblemente.

Como la modalidad de enseñanza guarda estrecha relación con el contenido a transmitir, no es de extrañar que en las zonas rurales, donde los niños desarrollan habilidades para el trabajo en el campo, tales como : trenzar un lazo, enrollar alambre, recolectar nueces o aceitunas, etc., la transmisión de los saberes propios para la 
realización de estas actividades se base en la demostración y la imitación de la acción, priorizándose aspectos visuales por sobre los auditivos.

Heredia (1996) afirma a propósito de lo señalado que "se produce algo así como una claudicación del discurso ante el lenguaje de la acción corporal y física y se pone también de manifiesto la prevalencia de lo físico y de lo motor, por sobre la reflexión o la explicación..” y que además “....el contexto de aprendizaje escolar es eminentemente verbal y que el niño se ve forzado a abandonar el estilo de aprendizaje vernáculo, basado en percepciones visuales y mímesis"(pp. 75).

Cuando el aprendizaje se refiere a conocimientos prácticos, tomando una característica descripta por Elliot (1983), quien se refiere a la ausencia de justificaciones para fundamentar por qué un procedimiento es correcto y otro no lo es, podemos pensar que en la escuela se suele alentar la reflexión sobre los procedimientos empleados por el alumno para la resolución de problemas, siendo de cierta manera extraño para el niño el cuestionamiento a un proceder adquirido como correcto.

Desde una vertiente de trabajo semejante, Ruth Paradise (1996) ha realizado investigaciones en México sobre cómo interviene en el aula escolar el conocimiento cultural de niños indígenas mazaguas

Conceptualiza el conocimiento cultural como "un tipo de conocimiento compartido con otros miembros de su grupo y que se vincula a su mundo socio-cultural cotidiano". Por medio de comportamientos y actitudes frente al trabajo escolar, este conocimiento aparece en el aula. Paradise alude a un rasgo común a diferentes grupos indígenas latinoamericanos, que es “...el fuerte énfasis de la observación como estrategia preferida para el aprendizaje. Dicha tendencia se manifiesta en cuestiones como la agudeza de la disposición perceptiva visual en términos de comportamientos, la autonomía y capacidad para la autodeterminación, la experimentación y el perfeccionamiento en el juego solitario de las destrezas que se están aprendiendo, la responsabilidad temprana en las actividades de trabajo ya aprendidas y la ausencia relativa de instrucción verbal y formulación de preguntas" (Paradise,1996).

Esta orientación a la observación, aparece en el aula en una amplia gama de interacciones no verbales en la relación con el maestro (quedarse sentados en silencio con las manos sobre las piernas y mirando, en los momentos en que están necesitando 
ayuda) y con los compañeros (observar el trabajo de los otros y cotejar con el propio o en búsqueda de claves que los orientan en una situación irresuelta).

A medida que avanzan en su estudio estos niños se van familiarizando más con las expectativas y pautas interaccionales de la escuela, como así también coordinando su accionar con el de los maestros que realizan su propio proceso de aprendizaje para decodificar adecuadamente los mensajes no verbales de los alumnos.

Otros aportes interesantes para pensar nuestra problemática son los de Carraher, Carraher y Schliemann (1991) quienes estudiaron el aprendizaje de las matemáticas en niños brasileños que trabajan en el mercado o la calle, y a partir de sus resultados cuestionan la noción de "privación cultural" utilizada frecuentemente para explicar las dificultades escolares de los niños de sectores carenciados.

A través de sus estudios, demuestran como los niños resuelven acertadamente diversos problemas matemáticos inherentes a las situaciones de transacción comercial que constituyen su cotidianeidad, mientras que tienen dificultades para resolver los mismos problemas en una prueba escolar. A la vez describen los razonamientos empleados por los niños para llegar a los resultados correctos (por ejemplo para cobrar de acuerdo al peso del producto vendido o dar el vuelto al cliente), y éstos difieren en muchas ocasiones de las reglas aritméticas que la escuela enseña.

También encuentran que al manejar objetos concretos y estar en una situación familiar, pueden resolver mentalmente (a veces en voz alta) diferentes operaciones, que les resultan más difíciles por escrito y en una situación hipotética.

Por lo tanto, lo que emerge es la coexistencia de diferentes modos para la resolución de problemas, unos ligados a las prácticas de subsistencia y aprendidos prácticamente por los niños ,y otros provistos por la escuela y pensados desde las disciplinas formales que constituyen la currícula escolar.

Si al enseñar matemáticas, el docente desconoce la lógica que el alumno emplea en sus actividades de subsistencia, probablemente genere un conflicto entre dos modalidades de razonamiento coexistentes, que se expresará como dificultad de aprendizaje y que, desde una perspectiva etnocentrista termine atribuyéndose a carencias del niño de condición humilde.

Si bien muchos de los aportes teóricos trabajados no se refieren específicamente a las pruebas de diagnóstico, hemos creído conveniente reflexionar sobre los mismos, en 
tanto nos ayudan a contextualizar las situaciones en la que se inserta la toma experimental del DIPUM, especialmente los enfoques antropológicos, que nos dan cuenta de cómo lo cultural incide en la relación educativa y en la percepción mutua de los actores que participan de ella.

Creemos que así, la discusión sobre la utilidad del DIPUM será mas provechosa.

\section{Método}

\section{Participantes}

La muestra estuvo conformada por un total de 68 niños, 37 de escuelas rurales y 31 de una escuelas urbano marginal.

La zona en la que trabajamos se encuentra en el Valle de Punilla, comprendiendo la comuna de Casa Grande (Esc. San Martín), la Pampa de Olaen (Esc. Obispo Salguero y Ramón Ramallo), Piedras Grandes (Esc. Alfonsina Storni) y el paraje La Punilla (Esc. Silvano Loza) aledaño a la localidad de Villa Giardino.

Podemos rescatar como aspectos comunes a las 5 escuelas: baja matricula, grados múltiples, directoras con grados a su cargo, servicio de comedor, la proximidad a zonas agrestes y con atractivo turístico, clima seco (salvo en verano) y amplias variaciones térmicas a lo largo del año.

En cuanto a actividades productivas de la zona, la agricultura y ganadería tienen escaso desarrollo, existiendo la cría de animales en pequeña escala, vinculada a la subsistencia familiar y a la comercialización informal.

Las escuelas se diferencian en cuanto al origen de las familias de los alumnos (lugareños o inmigrantes de ciudades), los niveles de instrucción de los padres, las actividades de subsistencia, el acceso a servicios de luz, agua y transporte, la proximidad a centros urbanos, el régimen de trabajo (media jornada, jornada completa y albergue) y la presencia (o ausencia ) de docentes de ramos especiales como Educación Física, Plástica y Tecnología y Tareas Rurales y de Jardines de Infantes.

\section{Instrumento}

El DIPUM fue concebido para su empleo en sectores urbano-marginales (Alderete 1995), ante la evidencia de que los instrumentos de evaluación de preescolares con los 
que se contaba, por su contenido y/o lenguaje, resultaban adecuados para los sectores medio-altos de la comunidad, pero sesgaban la medición de las competencias de niños residentes en barrios humildes o asentamientos villeros.

Este sesgo (asociado a la escasa validez conceptual de dichos instrumentos ) se podía superar en tanto se consideraran las características socio culturales de los niños al momento de diseñar los ítems que componen la prueba.

Para ello, se realizaron estudios de las comunidades que utilizan el servicio de las escuelas municipales de Córdoba, observándose algunas características de los procesos de socialización, los hábitos, costumbres, lenguaje y juegos, con el objetivo de adecuar los materiales y consignas que componen la prueba, a la experiencia cotidiana de los niños.

Para la selección de los objetivos a evaluar, se consideraron los correspondientes al Jardín de Infantes, la currícula de primer grado, aportes teóricos de los enfoques constructivistas, las opiniones de maestras de primer grado recopiladas mediante una encuesta y consultas a especialistas en Psicología Evolutiva, Educacional y Educación Física.

La versión del DIPUM que empleamos en nuestra investigación evalúa objetivos del Dominio Perceptivo Motor (Motricidad Global y Selectiva) y Cognoscitivo (Conocimiento Físico y Lógico ). Está compuesta por 22 ítems, de ejecución individual consistentes en tareas que el niño debe realizar, luego de una breve explicación y/o demostración del evaluador. La ejecución se califica en una escala de 0 a 2 según su adecuación a la consigna presentada. De este modo se obtienen puntuaciones para cada grupo de objetivos y dominios.

Los estudios confiabilidad realizados mostraron estabilidad en los puntajes (los coeficientes varían entre .70 y .92 para cada objetivo, para la prueba total el coeficiente encontrado es de .83) y consistencia interna (los coeficientes alfa encontrados varían entre .74 y .96, para cada objetivo, para la prueba total fue de .94). Del análisis de ítems se desprende que los índices de dificultad son cercanos a .80. Los correspondientes estudios de validez conceptual y contenido han arrojado también resultados satisfactorios. (Alderete,2001). 


\section{Procedimiento}

Realizamos encuentros de capacitación para la toma del DIPUM con las docentes, quienes participaron de la recolección de datos al comienzo del ciclo lectivo. Supervisamos las condiciones de administración para cuidar que no hubiera variaciones significativas en la forma de tomar la prueba.

Los ítems de Motricidad Global se evaluaron grupalmente y el resto en forma individual.

El trabajo sobre los diagnósticos llevó a un seguimiento más detallado de las áreas en que los niños exhibieron dificultades para apreciar si éstas aparecían en situaciones distintas a la de la prueba y si había o no progresos.

Las principales dificultades se observaron en Motricidad Selectiva, Clasificación, Correspondencia entre Conjuntos y Estructuración del Espacio. La mayoría de los niños no tuvieron dificultades en Motricidad Global, Conocimiento Físico y Seriación.

Las maestras seleccionaron actividades de aprendizaje que facilitaran la adquisición de las habilidades menos desarrolladas, intercambiando sugerencias (ver anexo, cuadro 1 y cuadro 2). Algunas de las actividades fueron semejantes a los ítems de la prueba (doblar y cortar papel, colorear figuras, etc.), rescatadas de las tradicionales prácticas de aprestamiento, o creadas por las docentes con elementos de la zona.

Resulta difícil estimar con precisión los niveles de avance de los alumnos y si estos son atribuibles a las actividades realizadas o no, pero para las docentes resultó provechoso atender de forma sistemática estas áreas del desarrollo.

\section{Análisis de Datos}

Se creyó conveniente identificar los objetivos específicos mejor logrados y aquellos que les resultaron más dificultosos en cada dominio evaluado, analizando las respuestas obtenidas por los niños en cada objetivo.

De las respuestas surge que los conductas más difíciles de lograr son: para el Dominio Perceptivo - Motor, en el área de Motricidad Global: Saltar coordinando movimientos de pies y brazos. En Motricidad Selectiva: Pintar una figura respetando los contornos de la misma, doblar papel y cortar papel por una línea de puntos. 
En el Dominio Cognoscitivo, para Clasificación: Identificar criterios de agrupamiento empleados por el evaluador y justificar verbalmente el agrupamiento realizado por el mismo niño. En Elaboración de conceptos numéricos: Mantener la correspondencia entre los elementos de los conjuntos al variar la distancia entre los mismos, y en Estructuración del espacio: Identificar las posiciones relativas "debajo de", “al costado de", “adelante y atrás de", empleando material concreto.

A fin de analizar la adecuación de la prueba para la evaluación de los niños pobres de zona rural, considerando que los estudios previos mostraron su validez para evaluar niños de zona urbano marginada, sometimos a prueba la hipótesis de que los grupos urbano - margianal y rural no presentarán diferencias significativas entre sí respecto de su rendimiento en la prueba.

Comparamos los puntajes de los niños de las escuelas rurales y los de la Escuela Roque Saenz Peña (donde asistían niños que habitaban en la villa de emergencia "El Pocito") de la Ciudad de Córdoba, del estudio realizado por San Millán y Pauletti (1993).

Utilizamos la prueba de la mediana como procedimiento estadístico no paramétrico que permite comparar tendencias centrales entre dos muestras independientes.

Los resultados obtenidos muestran que los niños de la Ciudad de Córdoba obtienen los puntajes superiores en la mayoría de las áreas evaluadas por la prueba, siendo éstas las áreas en que encontramos las diferencias más significativas.

Tabla 1. Diferencias entre grupos rurales y urbano marginales en las escalas del DIPUM

\begin{tabular}{lc}
\hline Área Evaluada & $\chi^{2}$ \\
\hline Puntaje Total & $13,1941^{* *}$ \\
Motricidad Selectiva & $11,2724 * *$ \\
Clasificación & $10,0192 * *$ \\
Elaboración de Conceptos Numéricos & $9,5296 * *$ \\
Conocimiento Lógico & $7,1735^{* *}$ \\
${ }^{*} \mathrm{p}<.05 . * * \mathrm{p}<.01$. &
\end{tabular}


Estos resultados indican que la hipótesis de semejanza entre los grupos no reúne evidencia suficiente para ser aceptada.

Nos preguntamos entonces si el rendimiento que en conjunto se presenta como más bajo para las escuelas rurales, refleja una distribución homogénea de las dificultades en el interior del grupo-muestra.

Comparando la cantidad de alumnos que superan o no los puntos de corte, a partir de los cuales se considera alcanzado cada grupo de objetivos o dominio, observamos algunas diferencias según sea la escuela a la que los alumnos asisten. Los objetivos de motricidad selectiva, clasificación y de conocimiento lógico, son los que introducen las mayores variaciones. Esto nos sugiere que la población rural presenta una diversidad importante en su interior.

\section{Validez Predictiva}

Utilizamos el índice de asociación predictiva (small Greek lambda) desarrollado por Goodman y Kruskal (1954), que permite estimar la probabilidad de error cuando intentamos predecir la categoría en que el sujeto estará incluido en una variable criterio (en este caso la calificación en las distintas asignaturas en el primer trimestre) conociendo la categoría que le corresponde en la variable predictora (el DIPUM).

El nivel de medición requerido es nominal por lo que transformamos los puntajes a categorías dicotómicas en base a puntos de corte preestablecidos, tomando como predictores los puntajes totales de la prueba, por dominio y por grupo de objetivos. 
Tabla 2. Coeficientes de asociación entre los puntajes del DIPUM y las calificaciones de los niños en el primer trimestre.

\begin{tabular}{lcccc}
\hline & \multicolumn{3}{c}{ Asignaturas } \\
\cline { 2 - 4 } Dipum & matemáticas & lengua escrita & ciencias naturales & tecnología \\
& & & y sociales &, 22222 \\
\hline puntaje total &, 05263 &, 04762 &, 00000 &, 11111 \\
dominio perceptivo motor &, 00000 &, 095241 &, 00000 &, 38889 \\
motricidad selectiva &, 00000 &, 00000 &, 00000 &, 16667 \\
dominio cognoscitivo &, 10526 &, 09524 &, 00000 &, 27778 \\
conocimiento lógico &, 00000 &, 04762 &, 00000 &, 00556 \\
clasificación &, 00000 &, 00000 &, 00000 &, 22222 \\
conceptos numéricos &, 00000 &, 00000 &, 00000 & \\
estructuración del espacio & & & &, 111 \\
\hline
\end{tabular}

La tabla precedente permite verificar que no hubo asociaciones significativas entre las variables predictoras y criterio en ningún caso.

\section{Discusión}

A partir de los resultados, intentamos comprender, tanto las diferencias existentes entre los grupos, como también la escasa capacidad predictiva que el DIPUM tuvo en este caso.

Revisamos nuestra información referente a juegos y actividades que los niños realizan (trabajo, quehaceres domésticos, etc.), escolariadad y ocupación de los padres, características de los lugares en que habitan, etc., con el objeto de reflexionar sobre los mismos y su posible relación con el desempeño de los niños en la prueba.

Los datos obtenidos en este trabajo nos indican que el DIPUM está reflejando diferencias realmente existentes entre los grupos que deben ser comprendidas en función del contexto del que los alumnos forman parte.

Si bien no podemos asegurar que las condiciones de administración del DIPUM han sido las mismas en nuestro caso y el del grupo urbano-marginal con quien hacemos 
la comparación, San Millán y Pauletti (1993) encontraron también que los resultados obtenidos por los niños de la Roque Saenz Peña eran mayores que los de una escuelasemi rural.

Pensamos que las diferencias tienen que ver con que los niños de la zona ruralserrana (fundamentalmente los que asisten a las escuelas de la Pampa de Olaen ), en sus procesos de socialización primaria, desarrollan habilidades vinculadas a las tareas que tanto ellos como sus familias realizan habitualmente y al empleo de modalidades lingüísticas particulares, y éstas son distintas a las que se construyen en el contexto urbano-marginal.

En este sentido coincidimos con diferentes estudios (Bixio y Heredia 1991) (Borsotti 1984) que han señalado el papel que juegan los estilos educativos familiares y comunitarios en el comportamiento de los niños en la escuela.

Para fundamentar lo expresado en párrafos anteriores, analizamos los distintos objetivos evaluados por el DIPUM.

En la Motricidad Global, realizan bien la mayoría de los ítems y lo atribuimos a que habitualmente juegan al aire libre, corren, saltan alambrados, arrojan piedras, enlazan terneros, suben a árboles, cruzan arroyos saltando de piedra en piedra, nadan, montan animales (caballos, burros), etc.

Sólo presentan dificultades en el 1er. ítem, que les requiere saltar coordinando movimientos de piernas y brazos, más propio de actividades gimnásticas que de sus juegos habituales.

La Motricidad Selectiva la emplean en el trenzado, la recolección de hierbas, los juegos con piedritas (payana), construyendo casas y caminos con piedras y palitos, etc.

En los ítems que requieren el manejo del lápiz, tijera y papel (instrumentos habituales en la escuela) presentan dificultades, atribuibles a un escaso contacto previo con el material. Probablemente, los mismos no son utilizados en las actividades cotidianas de sus familias y tengan poco incentivo para esas tareas por parte sus padres, en general con bajos niveles de escolaridad. Esto se combina con que en su escuela no cuentan con jardín de infantes. 
En cuanto a los ítems del Dominio Cognoscitivo, el buen desempeño en Conocimiento Físico se desprendería de la escasa complejidad de las actividades a realizar y la familiaridad con los materiales.

No obstante, podemos señalar algunas dificultades en el reconocimiento de colores que varios niños nombraban equivocadamente, aunque los emplearan correctamente como criterio de agrupamiento. Esto nos sugiere (más que algún defecto visual) que los niños reconocen y diferencian colores pero no están familiarizados con su denominación.

En cuanto al Conocimiento Lógico, cuando se les solicita clasificar cubiertos de tres tamaños y colores diferentes, debemos reconocer que estos elementos pueden ser extraños a algunos niños (de hecho las maestras señalan que a veces deben enseñarles cómo usarlos), y esto podría dificultar la ejecución de la actividad requerida por el examinador.

La conservación de la noción de correspondencia entre conjuntos al variar la distancia existente entre los elementos que los integran, es una tarea en la que las dificultades aparecerían en forma más generalizada en los niños de esta edad (6-7 años), independientemente de su contexto socio cultural. Esto debería ser revisado en la prueba.

Las dificultades observadas para describir algo (las características de un objeto), justificar un agrupamiento (en los ítems de clasificación) o en la correspondencia entre objetos (elaboración de conceptos numéricos) cuando se debe decir que hay que agregar una bombilla, y directamente la agregan, podrían estar relacionadas a que estos ítems requieren la verbalización de percepciones y acciones.

Consideramos que esta característica de los ítems puede generar dificultades, y estas dificultades pueden deberse a las diferencias entre los estilos educativos vernáculos y los de la institución escolar (Heredia, 1996, Paradise, 1996).

Probablemente, en los procesos de socialización en los que no hay una distinción muy precisa entre juego y trabajo, y en los que, mediante la imitación de los roles de los adultos, los niños aprenden a realizar muchas de las actividades que posibilitan la subsistencia, las estrategias empleadas preferentemente sean la demostración y observación. Cuando los adultos o hermanos mayores (frecuentemente a cargo del 
cuidado de los más chicos) les enseñan cómo hacer algo, se "lo muestran" obviando la explicación verbal del procedimiento o justificación lógica del mismo.

En síntesis, las áreas que en las que se observaron diferencias significativas entre los grupos son las que requieren el empleo de materiales típicamente escolares y formas verbales de comunicación para expresar pensamientos y fundamentar actos realizados (algo que se cultiva preferentemente en la escuela).

Es interesante señalar que los niños de una de las escuelas, en la mayoría de los objetivos tienen un mejor desempeño que los de las otras. Todos ellos asistieron a Jardín de Infantes, y sus padres, en muchos casos, tienen niveles de escolaridad secundarios ó terciarios. El estilo de agricultura de la zona está influenciado por concepciones ecológicas, se valora y practica el trabajo artesanal, y varias familias son migrantes de grandes ciudades que buscaron las serranías como nuevo lugar para vivir.

Entendemos que, el contexto familiar de estos niños está culturalmente más cercano al escolar (y al de las maestras), en comparación con los de la Pampa de Olaen (más de campo adentro), que suelen comenzar primer grado sin haber asistido al Jardín de infantes.

En una escuela con población más heterogénea, también lo es el desempeño de los niños en la prueba. Si bien hay algunos que proceden de zona rural y realizan tareas de cuidado de ganado, recolección de leña, etc., la mayoría lleva una vida más propia de lo urbano, con TV, bicicletas, video juegos, etc.

En cuanto a la escasa o nula capacidad que el DIPUM ha mostrado para predecir el rendimiento escolar en el primer trimestre, debemos reflexionar críticamente sobre el procedimiento que empleamos para su estudio.

La muestra fue muy pequeña (37 alumnos), sería conveniente ampliar el número de casos. Respecto de la variable criterio (las calificaciones), observamos escasa variabilidad, lo cual indica poca discriminación. Ya señalamos que no hubo casos de No Satisfactorio y además nos llama la atención que todos los niños de la muestra hayan tenido en Ciencias Naturales la misma nota que en Sociales.

El procedimiento por el que se califica a un niño en el primer trimestre de clases podría estar sesgado por consideraciones de orden socio afectivo (entiéndase el efecto motivador o desalentador de una nota para un niño que recién inicia la escuela), y tal vez no nos esté dando cuenta de progresos específicos en el aprendizaje. Además, son 
notas generadas por 4 maestras diferentes, en el marco de la relación que cada una tiene con sus respectivos alumnos y obedeciendo a criterios que no podemos suponer totalmente homogéneos.

Por consiguiente, es posible que el DIPUM no está prediciendo adecuadamente las calificaciones que la maestra colocará a los estudiantes. En este caso resultaría más conveniente diseñar un instrumento ad-hoc, estipulando los logros esperables en las diferentes áreas y aplicarlo en las escuelas de la muestra de un modo que garantice cierta homogeneidad de medición, y una definición operacional del rendimiento escolar más accesible a la contrastación

Un tercer aspecto a considerar es que trabajamos en base a los diagnósticos, para implementar actividades que ayudaran al desarrollo de las habilidades en las que detectamos mayor dificultad. Este es un factor interviniente que normalmente no está presente en el proceso educativo (y que debería controlarse al estimar la Validez Predictiva), e implica un seguimiento del alumno que puede incidir en su rendimiento.

Otro tema para la reflexión, tiene que ver con los supuestos de orden teórico que sustentan la relación entre los contenidos que integran la prueba que estamos estudiando y el aprendizaje escolar. Por ejemplo, esperábamos una asociación entre motricidad selectiva (como predictor) con lengua escrita (como criterio), pero reflexionando sobre los resultados y atendiendo a lo que se prioriza desde los enfoques imperantes en el área, de orientación Psicogenética, esta relación no es necesariamente esperable.

Podríamos suponer que el aprestamiento tiene una incidencia global sobre el rendimiento, es decir que se podría esperar una relación de predicción desde los puntajes totales, pero sobre esto tampoco hallamos evidencia.

Tal vez, podamos encontrar un panorama diferente si estimamos la capacidad de predicción a mediano plazo, es decir, considerando el rendimiento hacia fin de año. La apreciación final del rendimiento en un ciclo supone un mayor conocimiento del alumno por parte del docente, porque permite verificar si las dificultades que el niño pueda tener con las tareas escolares persisten en el tiempo o son superadas. Este proceder tiene la desventaja de que al aumentar el tiempo transcurrido entre las dos mediciones pueden influir sobre la variable criterio más cantidad factores 
Las diferencias estadísticamente significativas que hallamos entre los grupos urbano-marginal y rural al analizar sus rendimientos en la prueba, nos indican que la hipótesis de semejanza entre los grupos, no reúne evidencia para ser aceptada.

En base a los resultados obtenidos, evaluamos, en un primer momento, la posibilidad de reformular algunos ítems del DIPUM, buscando materiales más afines a la experiencia de los niños (arcilla, ramitas o tientos para trabajar motricidad selectiva, imágenes de animales en lugar de cubiertos para clasificar, objetos naturales para describir en lugar de un autito, etc) con el objetivo de elaborar una versión adaptada de la prueba. No obstante, fuimos desestimando esta línea de trabajo al advertir que al ampliar la gama de situaciones para evaluar los dominios en cuestión, nos alejamos progresivamente de lo que la escuela requerirá de sus alumnos.

Vale decir, el manejo de papel y lápiz y el empleo preferente de códigos verbales para la comunicación, resultan prácticamente irremplazables para la escuela rural actual, independientemente de que el niño subsista en base a la cría de animales, la recolección de hierbas o el trabajo de la tierra, y que en consecuencia posea habilidades y estilos de aprender funcionales a estas actividades.

Si la prueba debe ser acorde a los requerimientos de habilidades que las escuelas rurales establecen, el DIPUM está en condiciones de ser empleado, pues su contenido se corresponde con estos requisitos.

Muchas de las maestras así lo han señalado, descubriendo que al trabajar sobre la prueba y las actividades surgidas a partir del análisis de los diagnósticos, se reencontraron con las prácticas de aprestamiento implementadas durante años .

Si bien habían caído en un relativo desuso, por influencia de nuevos enfoques en política educativa, estas prácticas son la única alternativa con que cuentan las docentes para familiarizar al niño con el trabajo escolar, fundamentalmente cuando no han asistido a Jardín de Infantes y sus saberes provienen de una realidad distinta a la de la escuela.

Verbalizar el pensamiento y explicar porqué se ha obrado de tal o cual manera, proviene de una tradición educativa que prioriza los códigos lingüísticos elaborados y cierta lógica reflexiva y que no valora, al menos en el mismo nivel, un hacer vinculado a las necesidades prácticas, aprendido mediante la observación y experimentación y que 
se justifica en su eficacia para obtener lo que se busca, sin fundamentar necesariamente por qué se logró.

Mientras ésta tradición se mantenga, conocer la habilidad verbal de los niños resulta necesario y por lo tanto, quitar o reemplazar éste componente en el DIPUM sería un despropósito.

Podemos seguir sosteniendo el supuesto de que los niños de las zonas rurales en general poseen habilidades perceptivo - motrices y cognoscitivas semejantes a los de la zonas urbano-marginales, y que visualizarlas depende de que la situación de evaluación se adecue a sus contextos culturales y sus prácticas cotidianas; pero la Validez Conceptual del DIPUM, se fundamenta en que aceptemos las diferencias existentes entre los grupos, acotándolo al contexto particular de lo que hoy pretende la escuela que un niño sepa hacer.

Esto no significa que si ampliamos nuestra mirada a ámbitos diferentes del escolar (por ejemplo en los estilos de aprendizaje que se instrumentan para actividades de subsistencia) no encontremos otros resultados, donde podamos advertir cómo niños poco competentes en la escuela, son exitosos para resolver numerosos problemas inherentes a otro tipo de labores.

En síntesis: el DIPUM constituye un elemento útil de trabajo, en tanto interpretemos los resultados en directa relación con las tareas que se le proponen al niño, y sin realizar inferencias sobre lo normal ó deficitario de su desarrollo perceptivo motor ó cognoscitivo, ya que la gama de situaciones que componen la prueba (básicamente de actividades escolares) no son representativas de todas aquéllas en las que el niño podría poner en juego sus habilidades, para resolver problemas directamente vinculados a su subsistencia o entorno cotidiano.

El hecho de que estemos trabajando con una prueba del tipo "con referencia a criterio", guarda coherencia con la interpretación de los resultados que proponemos, ya que por su naturaleza éste tipo de instrumento sirve para obtener información respecto de la medida en que los sujetos realizan ciertos comportamientos claramente definidos y en una situación particular.

En tanto tengamos conciencia a de los alcances y límites de lo que llegamos a conocer a partir del uso del DIPUM, el mismo puede ser un recurso útil para trabajar en el mejoramiento de la tarea educativa. 
Nos queda por realizar otra discusión, que recién empezamos a vislumbrar, una vez avanzado el trabajo, la referente a la forma en que el docente reconoce, valora e instrumenta para el diseño de sus estrategias pedagógicas, lo que el alumno trae consigo al momento de llegar a la escuela. Conocer las formas en que los niños de zona rural desarrollan habilidades perceptivo-motrices y cognoscitivas, las emplean en las actividades de aprendizaje para la subsistencia, y descubrir la metodología particular para la transmisión de saberes y costumbres de sus comunidades, es una tarea que excede este trabajo. No obstante creemos necesario alentar estudios sobre estos aspectos, a fin de aprovechar éstos conocimientos en el mejoramiento de la calidad del servicio educativo que se brinda a estos sectores de la población.

Para ello se requiere de estrategias investigativas distintas y complementarias a las desarrolladas por nosotros, y talvez la etnografía sea una de las más adecuadas.

Reflexionando sobre la estrategia de transferencia de tecnología y capacitación del docente en servicio, creemos que el espacio compartido fue una instancia eficaz para incorporar nuevos conocimientos, pero también para revalorizar los construidos durante años en el trabajo áulico.

El hecho de que las maestras participaran de la indagación de los factores explicativos de los resultados hallados en el estudio, ha contribuido a la desmitificación de los tests, como instrumentos acabados y de valor absoluto y ha alentado en consecuencia, el cotejo de los datos con otras fuentes de información. 


\section{Referencias}

Alderete A.(1995). Elaboración de instrumento de diagnóstico de habilidades básicas para el aprendizaje en primer grado a ser utilizada en niños pertenecientes a grupos urbano-marginales. Informe presentado a la Secyt. UNC. Cba.(Inédito).

Alderete, A; Brussino, S.; Scherman, P. y Furlán, L. (2001). Diagnóstico de habilidades básicas para el ingreso a primer grado de niños pertenecientes a grupos urbanomarginados (DIPUM). Ponencia presentada al XXVIII Congreso Interamericano de Psicología. Santiago de Chile.

Bixio B.y Heredia L. (1991). Distancia Cultural y Lingüistica. Bs As: Centro Editor de AméricaLatina

Borsotti, C. (1984). Sociedad Rural Educación y Escuela en América Latina. Bs. As: Kapeluz

Carraher T., Carraher D. y Schliemann, A.(1995). En la vida diez en la escuela cero. México: Siglo XXI.

Ezpeleta J. (1991). Escuelas y Maestros. Condiciones del trabajo docente en la Argentina. Bs. As. Centro editor de América Latina.

Hays, W. (1966). Statistics for Psicologists. New York: Holt, Rinehart and Wiston.

Heredia, L. (1996). La organización local de las prácticas educativas en los medios desfavorecidos. Córdoba: Dirección General de Publicaciones U.N.C.

Himmel, E. (1979). Tendencias actuales en la evaluación del rendimiento escolar. Rev. de Tecnología Educativa, 5, (2), 171-194.

Paradise, R. (1996). El conocimiento cultural en el salón de clase: niños indígenas y su orientación hacia la observación. México: Documento DIE 33.

San Millán, M. y Pauletti, A. (1993). Trabajo Final de Licenciatura en Psicología. U.N.C.

Tedesco, J. C. (1981): Características y necesidades educativas de los niños, jóvenes y adultos en las poblaciones menos favorecidas, rurales y urbanas. Bogotá, 
Colombia: Documento para seminario de innovaciones en la formación y perfeccionamiento de educadores.

Unión de Educadores de la Provincia de Córdoba (1996). Proyecto Docentes y Escuelas Rurales de Córdoba Jorge W. Ábalos. 


\section{Anexo}

Cuadro 1. Actividades elaboradas por las maestras. Dominio Perceptivo Motor

\begin{tabular}{|c|c|}
\hline DOMINIO & PERCEPTIVO MOTOR \\
\hline $\begin{array}{l}\text { MOTRICIDAD } \\
\text { GLOBAL } \\
\text { Estas actividades han } \\
\text { sido desarrolladas en } \\
\text { los horarios de } \\
\text { Educación Física y } \\
\text { durante los recreos. }\end{array}$ & $\begin{array}{l}\text { - Lanzar y recibir una bolsita con arena, en parejas y en grupos. } \\
\text { Sobre esta actividad se realizan variaciones en la distancia de } \\
\text { lanzamiento, la mano utilizada y se practican jugos como "el } \\
\text { distraído" } \\
\text { - Saltar en uno y otro pie y con pies juntos, empleando cubiertas } \\
\text { en desuso y hacia adelante o atrás. Un juego muy empleado } \\
\text { fue la rayuela y las carreras con obstáculos } \\
\text { - Saltar imitando animales (ranas, canguros, etc.), con sogas en } \\
\text { el suelo que marcan distancias y se separan progresivamente. } \\
\text { - Marcar diferentes ritmos caminando, trotando, corriendo y } \\
\text { - Laltando. } \\
\text { - Canzar pelotas para embocar en el aro, jugar al delegado } \\
\text { - Caminar sobre una soga y saltarla de diferentes formas. } \\
\text { - Danzas folclóricas para trabajar ritmos y coordinación de } \\
\text { movimientos amplios. } \\
\text { cuerabajos de reconocimiento de lateralidad con el propio }\end{array}$ \\
\hline $\begin{array}{l}\text { MOTRICIDAD } \\
\text { SELECTIVA } \\
\text { Estas actividades han } \\
\text { sido trabajadas en } \\
\text { Plástica y } \\
\text { Tecnología como así } \\
\text { también integradas } \\
\text { en Lengua, } \\
\text { Matemáticas y } \\
\text { Ciencias }\end{array}$ & $\begin{array}{l}\text { - Con papel: recortes siguiendo línea punteada y de letras, } \\
\text { palabras o dibujos-fotos; plegado simple y de mayor dificultad } \\
\text { para armar barcos, aviones, etc. } \\
\text { - Para manejo de lápiz además de los que se usan en escritura se } \\
\text { hicieron dibujos y coloreado de figuras } \\
\text { - Trabajo con un punzón, trazando líneas y punteados } \\
\text { - Con elementos naturales se hicieron collages, construcción de } \\
\text { nidos, trenzados, pegado de semillas en los lápices. } \\
\text { - Coloreado, pegado, recorte y armado de figuras sobre papel y } \\
\text { cartulina. } \\
\text { - Con recortes de cartón y maderas se armaron herramientas } \\
\text { - simples } \\
\text { El juego de la payana es practicado habitualmente por los } \\
\text { niños, como así también el uso de la hondera } \\
\text { - Armado de rompecabezas. }\end{array}$ \\
\hline
\end{tabular}


Cuadro 2. Actividades elaboradas por las maestras. Dominio Cognoscitivo

\begin{tabular}{|c|c|}
\hline DOMINIO & COGNOSCITIVO \\
\hline $\begin{array}{l}\text { CONOCIMIENTO } \\
\text { FISICO } \\
\text { Actividades } \\
\text { realizadas en } \\
\text { Ciencias Naturales y } \\
\text { Lengua oral y escrita }\end{array}$ & $\begin{array}{l}\text { - Observación diaria de las condiciones climáticas } \\
\text { - Observación y descripción de objetos y elementos del entorno, } \\
\text { tanto vegetales como animales, considerando su tamaño, color, } \\
\text { utilidad, etc. } \\
\text { - Observación y manipulación de objetos de uso cotidiano, } \\
\text { descubriendo su modo de funcionamiento y características }\end{array}$ \\
\hline $\begin{array}{l}\text { CONOCIMIENTO } \\
\text { LOGICO } \\
\text { Clasificación }\end{array}$ & $\begin{array}{l}\text { - Trabajo con los bloques Diennes, agrupando figuras según su } \\
\text { color, tamaño, espesor y forma. } \\
\text { - Agrupamiento de elementos naturales en seres vivos y no } \\
\text { vivos, vegetales y animales, comestibles y no comestibles. } \\
\text { Reconocimiento de las especies vegetales como medicinales, } \\
\text { venenosas, aromáticas, etc. } \\
\text { - Formar filas del más alto al más bajo y viceversa } \\
\text { - Ordenar tiras de cartulina de la más larga a la más corta y } \\
\text { viceversa } \\
\text { - Ordenamiento de objetos de más grande a más chico y } \\
\text { viceversa }\end{array}$ \\
\hline $\begin{array}{l}\text { Elaboración de } \\
\text { Conceptos } \\
\text { Numéricos }\end{array}$ & $\begin{array}{l}\text { - Correspondencia entre números y material concreto } \\
\text { - Correspondencia término a término: Entrega de la mochila } \\
\text { respectiva o un lápiz a cada compañero. } \\
\text { - Correspondencia bi-unívoca con tizas de diferente color } \\
\text { - Equivalencia numérica, colocando tantas tizas de un color } \\
\text { como las que hay de otro. } \\
\text { - Conservación de cantidades de líquido en recipientes de } \\
\text { diferente tamaño } \\
\text { - Colocar bolsitas de arena dentro, fuera, al costado, arriba, } \\
\text { - Ubajo, delante, atrás de cubiertas de auto, cajas, etc. } \\
\text { - Ubicarse en las diferentes posiciones en relación a un referente } \\
\text { - Ubicarse a la derecha o izquierda de un compañero } \\
\text { - esconderse }\end{array}$ \\
\hline
\end{tabular}

